\title{
Analytic system to evaluate efficient driving programs in professional fleets
}

\author{
Laura Pozueco, Alejandro G. Tuero, Alejandro G. Pañeda, Xabiel G. Pañeda, David Melendi, \\ Member, IEEE, Roberto García, Gabriel Díaz Orueta, Senior Member, IEEE and Abel Rionda
}

\begin{abstract}
Interest in energy cost saving and in global warming have persuaded transport companies to apply measures to reduce fuel consumption. Efficient driving is one of the most employed solutions as it allows savings in fuel consumption of around $10 \%$ with a minimal investment. The drawback is that efficient driving is a learning process and it greatly depends on the drivers' behavior, which in turn is closely related to their motivation. If drivers are not really involved or after some time their interest decreases, efficiency improvements would disappear. Thus, an efficient driving program should make drivers' motivation one of the main targets. One option could be the implementation of reward programs. However, these should be based on a clear individual evaluation process, as an unfair system could lead to discomfort, complaints and repudiation. In this paper, we propose an analytic system, based on the detection of efficient and inefficient behavioral patterns, to evaluate the individual driver's progression in efficient driving with the aim of being the basis of a reward program. The system receives relevant, driving related, vehicle information every 1.5 seconds, allowing a precise searching of patterns. It has been tested successfully in 16 bus companies, analyzing the performance of $\mathbf{8 8 0}$ professional drivers. To accurately illustrate the analytic process, three detailed driver analyses have been included as a case study. Results of this applied research on the eco driving field show that the proposed system identifies efficient and inefficient actions that are used to fairly evaluate the drivers' performance.
\end{abstract}

Index Terms - Efficient driving, professional driver evaluation, analytic system, fuel savings, public transportation

\section{INTRODUCTION}

$\mathrm{R}^{\mathrm{o}}$ OAD transportation is one of the most important $\mathrm{CO}_{2}$ producers and its emissions are partially responsible for global warming [1]. Conscious of this environmental problem, the European Commission has established the goal of limiting the increase of $\mathrm{CO}_{2}$ transport emissions to $8 \%$ above the 1990 level by 2030 [2]. Governments and companies have tried to revert this situation implementing different types of measures. In some cases, the actions have been oriented to improve vehicle technology and in others to make a better use of that already existing, for instance, by selecting the best routes,

Manuscript received. This work was supported by the Spanish National Research Program within the project TIN2013-41749-R, the Asturian Research Programme (PCTI) with the project GRUPIN-14-065 and the General Direction of Traffic (DGT) of Spain through the project SPIP20141277.

L. Pozueco, A.G. Tuero, X.G. Pañeda, D. Melendi and R. García are with the Informatics Department, University of Oviedo, Gijón, Spain (e-mail: \{pozuecolaura, garciatalejandro, xabiel, melendi, garciaroberto\} @ uniovi.es). applying predictive maintenance or driving more efficiently.

Driving efficiency has been promoted through different governmental programs around the globe. One of the main reasons is that these programs can be applied to any type of vehicle, new or old, and the investment is much reduced. Moreover, following eco-driving recommendations, fuel consumption can be decreased by around $10 \%$ [3] which is a very significant reduction. However, efficient driving is a lifelong learning process that requires a constant update throughout the driver's working life. In the last few years, efficient driving programs have consisted of a combination of different activities such as face-to-face courses, training, onboard assistance, etc. Many of these activities have demonstrated their capacity to produce short-term improvements. Using a combination of them, program designers try to maintain these efficiency improvements in the long term. However, long-term efficiency relies heavily on the drivers' motivation and involvement. The lack of these factors gradually reduces the early improvements [4].

One way to promote drivers' motivation is the establishment of reward programs which make them co-beneficiaries of their collective effort. Nevertheless, to support a fair process, the definition of a clear objective and unbiased evaluation method is necessary. This method should be supported by an analytic system which accurately evaluates individual driver performance.

In this paper, we present an analytic system which evaluates the drivers' individual performance. Based on the detection of efficient and inefficient behavioral patterns, the system is able to detect the driver's evolution from the efficiency point of view. Driving patterns are extracted from the information gathered from the Engine Control Unit (ECU) every 1.5 seconds. This information includes data related to speed, rpms or acceleration among others. Therefore, with our analytic system, the benefits for the drivers are twofold: on the one hand, the drivers receive personalized recommendations to improve their efficiency. On the other hand, they are evaluated and analyzed based on their driving behavior and not on biased

A.G. Tuero is Student at PhD program of Industrial Technologies at U.N.E.D

A.G. Pañeda and A. Rionda are with ADN Mobile Solutions, Gijón, Spain (e-mail: \{alejandro.garcia, abel.rionda\}@adnmobilesolutions.com).

G. Díaz is with the Electrical and Computing Engineering Department, Spanish University for Distance Education (U.N.E.D), Madrid, Spain (e-mail: gdiaz@ieec.uned.es). 
information such as fuel consumption. As a result, the motivation of the drivers to continue applying efficient driving techniques are maintained and the information produced could be used as the basis of an objective reward program and to support the trainer in the definition of the driver's instructional path. Combined with a learning methodology, such as [5], it could conform the basis of an efficient driving program.

In addition, the analytic system based on driving patterns allows us to analyze the driver's behavior in a continuous improvement process, which facilitates the incorporation of efficient driving behavior during the whole working day. With this approach, the driver would become efficient in any road or traffic conditions in the long-term, as the efficient behavior while driving should be performed under any circumstances, especially by professional drivers.

The system has been tested successfully in 16 companies in Spain and Morocco where 880 drivers were evaluated using it. Results of these real deployments of the system in different bus companies confirm its usefulness in driver evaluation as part of a general efficient driving program.

The rest of the paper is organized as follows: related work is outlined in Section II. Section III introduces the context where the system can be integrated and why it is important in efficient driving programs. Section IV details the analytic process which supports the system design. Section V describes the analytic system architecture and modules. Section VI includes a case study where three drivers were selected to show a detailed analysis. Finally, conclusions and future work are highlighted in Section VII.

\section{RELATED WORK}

Reduction in fuel consumption can be achieved through different approaches, such as predictive maintenance, fleet renovation or efficient driving training. However, due to operational and cost reasons, promoting eco-driving among drivers is a promising option to reduce emissions and fuel consumption and produce safety benefits [6].

The classical way to improve efficiency is to implement efficient driving courses. These courses can improve driver behavior and reduce fuel consumption in the short-term [7]. Another possibility is the use of simulators to consolidate knowledge on efficient driving after receiving a course. Authors in [8] conclude that this learning method can achieve significant reductions in fuel consumption, thus presenting an alternative to classical learning methodologies.

Particularly important is the training of professional drivers, as they generally drive longer distances, and furthermore, the transport sector is one of the greatest contributors of greenhouse gas emissions. Zarkadoula et al. [9] developed a pilot program in Greece with the aim of analyzing the results of eco-driving courses for urban bus drivers. Results showed a fuel saving of around $10 \%$. Similar studies have also been conducted in Sweden [10] and Canada [11] with positive results. Authors in [12] use a continuous monitoring system to obtain real-time feedback with information related to speed and acceleration measurements expressed in terms of "driver's driving style". When applied to a test fleet of 15 light commercial vehicles, a reduction of $7.61 \%$ of fuel consumption is obtained. They claim that this saving was achieved by encouraging drivers to apply a set of recommendations based on efficient driving. Nevertheless, drivers often revert to their original habits over time [13], highlighting that these approaches have several drawbacks, closely related with the end of the efficient driving programs and their non-obligatory nature.

The establishment of reward programs is a good idea to maintain the motivation of drivers, as seen in [14], where a method for utilizing fuel consumption data in an incentive system was developed successfully for a transport company. Harvey et al. [15] provide quantitative proof for including these incentives. However, to implement reliable reward programs, it is necessary to define a method of individualized evaluation as objective as possible, based on clear metrics and taking into account distortions due to other factors that may influence the performance of the vehicle. To that end, a complete analysis of the individual efficient driving process has to be carried out.

Until now, evaluation of an efficient driving process has been based mainly on consumption [9], [16] and there are no assessments of driving processes analyzed in depth. A system based exclusively on fuel consumption is insufficient to determine the improvement in driving, due to the multiple factors that affect the evaluation context, such as vehicle maintenance, weather or traffic congestion [17]. Other papers [18], [19] introduce some other indicators to calculate efficiency, such as acceleration, speed, revolutions, congestion or the weight of the vehicle. However, they only present basic reports in a short period of time. Moreover, their analytical systems are constrained to present the evolution of some metrics, such as braking and accelerations [20], without focusing on detecting inefficient driving behaviors. On the contrary, our analytic system to evaluate efficient driving presents a novel approach, since it is based on complex driving patterns that characterize the driver's behavior.

Only recently, some studies have started to introduce the analysis of driving patterns. In [21], using the vehicle telemetry, the system detects inefficient areas on a route, warning the user to adjust speed. However, results are based only on consumption; their system is not designed for a learning process and, as a consequence, it does not allow the establishment of an evolution in the efficient driving learning process. Thus, a clear individual evaluation process is an open issue. In this paper we design and implement a fair evaluation process for efficient driving using driving patterns obtained from the data collected during the driving process. Authors in [22] also based their proposal on information gathered from vehicles. However, their interval duration between two consecutive samples is too long to obtain accurate results. In addition, their analysis is based on the factors which most influence fuel consumption. Our proposal is based on data gathered from the vehicles with a high sampling rate and we transform this information into accurate driving patterns.

Our main contributions are the design and implementation of a learning analytic system, based on the recognition of driving patterns to evaluate individual driver performance and not on statistical summaries. Our solution is based on complex patterns 
from data collected every 1.5 seconds from the vehicles to detect inefficient situations, thus making it more accurate than other proposals. In addition, the proposed system has been deployed in 16 different professional fleets of urban public transport companies with 880 professional drivers and the studies have been conducted over long periods of time, which vary from a pilot program of eight months (since 2015) to a period of more than three years that is still ongoing today. Furthermore, to the best of our knowledge, there are no concise proposals to evaluate the individual driver's evolution in terms of efficiency and taking into account the characteristics of the driving context.

\section{BACKGROUND}

In our previous research, we developed a methodology to learn how to drive vehicles efficiently [5]. The methodology was based on the blended learning paradigm (using several learning tools such as an on-board system which provides realtime feedback and recommendations and face-to-face courses based on theoretical sessions and individual practical training) to be applied while the drivers are working. Therefore, we created a complex on-the-job training process with the aim of improving the efficient driving results in the long-term.

On the one hand, professional drivers attend theoretical courses, in which an expert in efficient driving gives the basic recommendations to achieve more ecological and economic driving. Fig. 1a shows an example of one of these sessions.

On the other hand, these theoretical courses are reinforced with practical sessions, in which professional drivers perform a complete route without advice to obtain the reference and then, on the same route, an expert trainer helps drivers with technical advice to apply the learned concepts in efficient driving (Fig. 1b). After that, performance comparisons between the reference route and the guided route are carried out in a Tablet to check the improvements in terms of efficiency and the maintenance of the time of the service (Fig. 1b).

The on-board feedback device is the key element in the learning process. It is composed of two elements: a monitoring device to gather data from the Engine Control Unit and an application to show recommendations and alarms while driving related to efficient driving behaviors [5]. Recommendations and alarms are based on basic parameters related to the efficient driving closely associated to the mechanical characteristics of the vehicles (such as speed, rpms, acceleration and instantaneous fuel consumption), which notify isolated events whose values exceed the maximum values established to consider driving safe and efficient. The recommendations and alarms are presented both visually and acoustically, with the aim of facilitating the incorporation of efficient driving techniques during the work activity (Fig. 1c). In addition, the feasibility of such feedback elements is evaluated in [23].

The learning methodology has been applied by our partner company, ADN Mobile Solutions, to all of its clients since 2012. This includes 16 transport companies and 880 drivers. Since the beginning of our cooperation, all the drivers have received a monthly report with indicators about their performance. Originally, the main performance indicator to evaluate the drivers' improvement was fuel consumption. This is the classic approach used in the majority of previous works [9],[10],[11]. However, fuel consumption is influenced by an important set of elements beyond the control of the driver, such as the weather or road conditions, state of the tires, etc. Thus, this approach is not very accurate. Considering this problem, we started to analyze other types of evaluations. Exams or specific tests were rejected, as they evaluate the driver's knowledge and not how this knowledge is really applied. Accordingly, our focus was to evaluate the behavior of the drivers while they were working, which actually reflects their real performance. We considered that the optimum approach was to infer the behavior of the drivers using information gathered from the vehicles. Thus we could compare this behavior with some well-known efficient and inefficient patterns. This new approach has been part of our feedback system since 2014 .

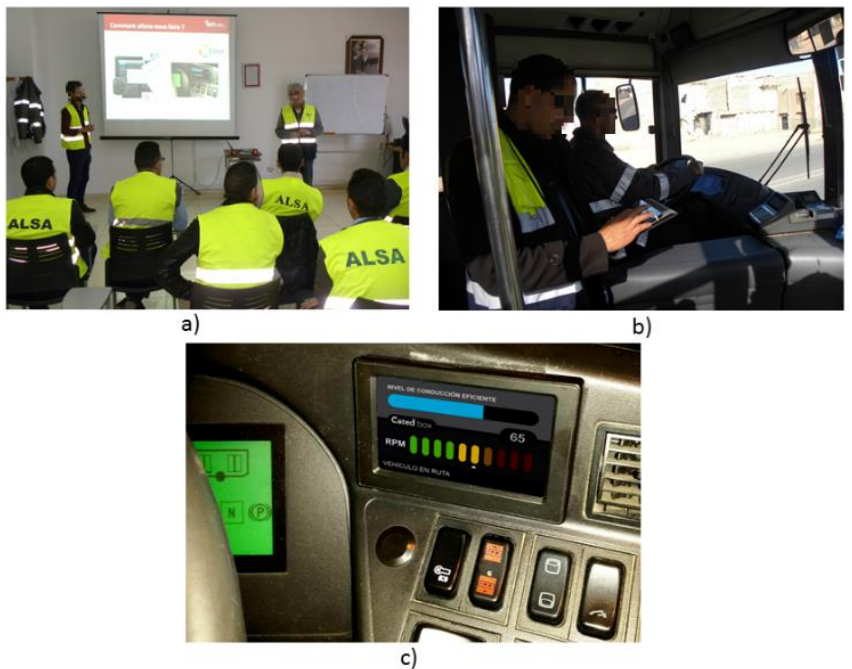

Fig. 1 a) Theoretical course in efficient driving b) Practical course in efficient driving c) On-board system to assist drivers in efficient driving

\section{ANALYTIC PROCESS}

In this context, we designed a learning evaluation system which uses as a source of information, data gathered from the ECU (Engine Control Unit) and some additional elements, such as a GPS receiver. The gathered information is stored in a nonrelational database and then combined with summarized information related to numerical identifiers of vehicles and drivers. Then, all the information is stored in an SQL relational database to process and extract driving patterns. From these data, the driver's behavior is extracted, which is directly related to efficiency and safety while driving. This system would not interfere with the drivers' normal activity and would reflect how they apply the efficient driving techniques.

Before describing in detail the analytic system, the following sections will summarize the analytical process. The analytic process is organized in four phases (Fig. 2) where the context is established, the baseline and the periodic analysis are calculated, and the learning progress is evaluated. All these phases shown in Fig.2 will be detailed later in Section C. The whole system is based on the detection of efficiencies and 
inefficiencies. To that end, some patterns which reflect these two types of behavior have been characterized. The patterns are combinations of values detected in the traces recovered from the vehicle, which reflect the application or not of an efficient driving technique. With our driving patterns, the influence of external factors on classical parameters such as fuel consumption are avoided and the evaluation is made taking into account how the drivers apply the efficient driving techniques learned during the face-to-face seminars throughout the working day.

The results will be used twofold. On the one hand, the trainers will have a tool to monitor driver progress and apply personalized training programs [24]. On the other hand, the person in charge of the fleet can use this information to implement a reward program which increases driver motivation. In addition, the learning analytic process will provide all the necessary information to construct the feedback report with personalized recommendations and evaluation results for each driver.

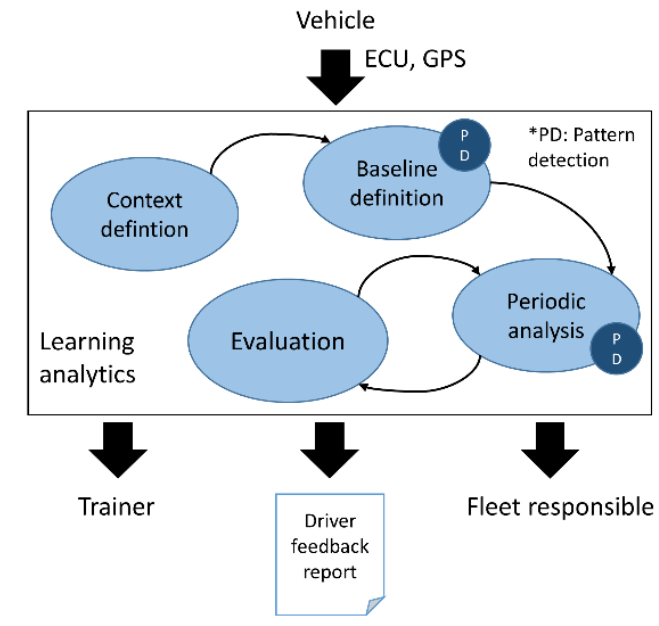

Fig. 2. Learning analytic process

\section{A. Information sources}

The analytic process starts with the collection of data from the vehicle driven by the driver who is going to be evaluated. This information, gathered by an on-board device, mainly comes from the ECU. To complement the information provided by the vehicle, the on-board system itself adds other important data such as the GPS position and $\mathrm{g}$ forces detected with an accelerometer. At this point, raw data is associated with a driver by a numeric identifier.

A trace with all of this information is composed every 1.5 seconds (with average values of the samples calculated during 1.5 seconds), transmitted out of the vehicle and stored to conform a huge information system with the vehicle performance. The components included in a trace are:

- Vehicle id: identifies the vehicle associated with the collected data.

- Timestamp: identifies the date and time of the day when the registered events have occurred, with the following format YYYY-MM-dd hh:mm:ss.xxx.
- Speed of the vehicle: average speed of the vehicle in $\mathrm{Km} / \mathrm{h}$.

- RPMs: average of revolutions per minute of the engine.

- Selected gear: current engaged gear.

- Acceleration: average acceleration of the vehicle in $\mathrm{m} / \mathrm{s}^{2}$.

- Accelerator pedal position: position of the accelerator pedal, measured in percentage.

- Brake switch: identifies whether the brake pedal is in use.

- Instantaneous fuel consumption: instantaneous fuel consumption of the vehicle in $1 / h$.

- Latitude: GPS latitude coordinate.

- Longitude: GPS longitude coordinate.

\section{B. Efficiency and inefficiency patterns}

To extract the driver behavior from the vehicle performance, we have designed a set of patterns which represent efficient or inefficient actions of the driver. These behavioral patterns are detected analyzing the traces recovered from the vehicle. Efficiency is represented by the "Inertia" pattern, while the other three show inefficient habits, which we call "Acceleration-Brake", "Brake-Acceleration" and "Idling". We made a brief introduction to the efficiency and inefficiency patterns as a base of an adaptive learning system in [25]. The described patterns complement the learning evaluation process in conjunction with the analytic system.

To be able to analyze the patterns we will define the Key Performance Indicators (KPI) [26]. This is a common term used for describing a performance measurement, being a tool to evaluate the success of a particular activity.

1) Inertia

Inertia is the basic efficiency pattern. It shows when the vehicle under study is running with zero fuel consumption. The conditions for this pattern are that the speed must not be zero, a gear (any) has to be engaged and the driver's foot has to be off the accelerator. According to this, the parameters set to find the pattern will be non-zero speed and null fuel consumption (this involves the gear and accelerator assumptions).

speed $\neq 0 ;$ fuel consumption $=0$

If the vehicle under study does not provide the consumption data, we will change it for the engaged gear (being different to zero) and accelerator (being 0\%) option.

speed $\neq 0 ;$ gear $\neq 0$; accelerator $=0$

This second option, which is $100 \%$ reliable, is not as common as the first. The reason is that the consumption data is found more regularly than the accelerator percentage.

The KPI for the inertia pattern will be its time percentage in relation to the total duration of the studied period, as well as the total change of speed which will be obtained from the difference between the initial and final speed.

\section{2) Acceleration-Brake $(A B)$}

The $A B$ pattern characterizes the abuse of the accelerator and brakes. It shows a lack of efficiency concepts, as the driver keeps speed constant by accelerating softly and then comes to a halt by braking progressively, instead of using inertia.

We look for periods where the driver accelerates permanently, immediately followed by the use of the brakes within a 2 second gap (as the driver uses the same foot for both pedals and has to change). 


$$
a>0 \rightarrow(t \leq 2 s) \rightarrow \text { brakes }=1
$$

If we do not have the "brakes on-off" data, we will substitute it by a negative acceleration, trying to distinguish the natural decelerations of the vehicle due to friction and motor retention. For this, we will pre-set a limit value as a pattern launcher.

$$
a>0 \rightarrow(t \leq 2 s) \rightarrow a \leq \text { limit } m / s^{2}
$$

We will record how many times this pattern happens every $100 \mathrm{~km}$ in the studied period, as a KPI. We will also save the duration of the pattern, and initial and final speed to obtain the total speed differential.

\section{3) Brake-Acceleration (BA)}

The aim of this pattern is to detect when the driver fails to keep the safety distance, which is both unsafe and inefficient. The BA pattern starts when an elevated intensity of deceleration occurs (which we identify with a harsh-braking event). After the harsh braking, the driver continues accelerating the vehicle. Then, the purpose of the harsh braking was not to stop the vehicle. For that reason, the BA pattern is related to anticipation while driving. The driver loses a great amount of the energy provided to the vehicle compared with when the safety distance has been maintained, so enabling the use of inertia.

We will dismiss every use of the brakes with the intention of stopping the vehicle, while we will pursue the hard braking situations intended to produce an immediate change in speed but without the will of stopping. The interpretation is that, by not maintaining the safety distance, the driver could not anticipate what the vehicle in front of him was going to do, so a brake event over the limits has to be done to avoid a collision.

In this case, we will look for the intensity of deceleration, using the same prefixed value used in the previous pattern. Nevertheless, it is necessary for the vehicle not to stop before accelerating for the pattern to be fulfilled.

$$
a \leq \text { limit } m / s^{2} \rightarrow\left\{\begin{array}{l}
a<0 \\
v \neq 0
\end{array} \rightarrow a>0\right.
$$

We will record how many times this pattern happens every $100 \mathrm{~km}$ as a KPI. We will check how long the pattern is and initial and final speed to see how much it decreases.

\section{4) Idling (Id)}

Traditionally, idling has been considered part of the set of recommendations to achieve more efficient driving as it has a great influence on fuel consumption. Therefore, idling is considered as an inefficient driving pattern and it makes sense in conjunction with the rest of our driving patterns, with the aim of refining the evaluation results and giving personalized recommendations. We try to detect periods over a certain amount of time where the engine is running but the vehicle is stationary. We have taken this approach but with special considerations to take into account the inherent characteristics of the transport service in order not to jeopardize the driver's evaluation results in efficient driving. The amount of time that sets the limit of the pattern has to be agreed with the fleet manager according to the needs of the service.

$$
v=0(t \geq \text { limits })
$$

In the case of the idling behavior, the KPI will be the time in percentage $(\%)$ of the total driving time for the studied period.

\section{Phases of the Learning Analytic Process}

To evaluate the driver's progress in accordance with the described efficient and inefficient patterns, we propose a learning analytic process with 4 different phases (Fig. 2). Thus, our evaluation of driving efficiency is based on driving patterns and their evolution over time. Therefore, the elements that condition the efficiency level and are closely related to fuel consumption such as the load of the vehicle or the state of the tires do not influence the efficiency results, as we evaluate driving behavior.

The first phase of the analytical process, context definition, is dedicated to obtaining the detailed description of all the aspects relevant to detect efficient driving patterns. Therefore, the context definition phase includes the identification of the type of service provided, the type of vehicle and its main characteristics and finally, the amount and type of data that it is possible to gather from the vehicle. These elements are closely related with the set of external factors that affect the efficient driving process and thus, the final evaluation results. We refer to type of service as the characteristics of the main purpose of the fleet and we differentiate between urban public transport, interurban passenger service and freight transport.

Relating to the type of service, there are great differences between urban public transport, a regular coach or a long distance coach traveling along the motorway. The first stops every few meters, the second in every town and the last only in major cities. The situations where the driver has to be efficient are very different. For that reason, the characteristic idling periods will also be different. Likewise, there are also differences between passenger service and freight transport. The time of the average idling period is used to characterize the type of service.

The context definition phase is also characterized by the type of vehicle (including simple buses, articulated buses, minibuses, trucks or solid urban waste trucks, among others). Another key element in this phase is the type of gearbox (automatic or manual) and the characteristic gearshift points.

Moreover, to complete the context definition phase, an evaluation of available data obtained from the vehicle should be carried out, with the aim of determining the quantity and quality of the obtained results of the evaluation process. The possibility of collecting and storing additional data implies differences in the definition of the patterns, as discussed in Section IV.B. For instance, the availability of information relating to the brake pedal provides more detailed information for pattern analysis.

The baseline definition phase states the driver's efficiency starting point. It is specific for each driver and establishes the details of the efficient driving behavior that will be used as the comparison factor to monitor the evolution of the driver's competence. Therefore, each driver has their own baseline, and the analysis of the learning progress is carried out based on their individual evolution in applying the efficient driving techniques. During the baseline phase, the driver's behavior is monitored and no feedback information is provided. The default duration period to collect data to calculate driving patterns for the baseline is established at one month. These patterns are characterized by a set of KPIs which will be used to produce general indicators to summarize the whole period.

The details of the tasks in the third phase, the periodic 
analysis phase, are the same as in the baseline definition, carrying out pattern extraction by analyzing all the data, but in different time periods. The periodic analysis phase (third phase) is carried out every month after the baseline definition phase. However, during the periodic analysis phase, drivers are assisted by the on-board device which provides real-time feedback related to recommendations of speed and rpms and alerts of harsh braking or acceleration events to help drivers apply efficient driving techniques. The purpose of the real-time recommendations is twofold: on the one hand, the feedback device maintains concentration on efficient driving throughout the whole working day. On the other hand, it permits long-term learning as the new driving habits are included little by little in the daily routine. Notwithstanding, the feedback device is focused on localized events. These are enriched with the complete analysis of the driving behavior, carried out during the third phase of the learning analysis process. Each of our designed driving patterns (Section IV.B) is analyzed every month and expressed in terms of its performance indicators (KPIs).

With the aim of determining the learning progress, the last phase (learning evaluation) compares all the indicators extracted in the baseline and the periodic analysis phases. This comparison allows us to see in detail how the driver is applying the efficient driving techniques over time. Therefore, for each driver, the driving performance, in terms of driving patterns, is compared from the baseline period to the following months. A positive evolution in driving efficiency is characterized by a reduction in the KPI indicators of the idle, AB and BA patterns, and an increment in the inertia KPI. Otherwise, the driver's performance in terms of driving efficiency is not evolving adequately. To complement this information and also to explain the origin of this performance, correlations between patterns, time (day of the week, hour of the day, etc.) and locations are analyzed. Thus, a deeper analysis can be carried out based on the day of the week, the hour or even the geolocation with the aim of focalizing the efficient driving recommendations in such elements that need to be improved. Consequently, personalized recommendations can be made in order to eliminate inefficiencies while driving in successive iterations of the learning process. All the processed information is used to complete a detailed report, with personalized information for each driver.

\section{ANALYTIC SYSTEM}

The analytic system has been developed based on a set of hardware and software elements that support the proposed methodology. Such software and hardware elements are grouped to build functional modules that perform specific tasks as part of the learning analytic methodology. All the necessary data to feed the analytical system are provided by the CatedBox system described in [5], which is a corporate operational system for companies interested in controlling their driving efficiency.

Fig. 3 shows the schema of the proposed system. To build the different modules of the analytic system, we previously load all the necessary data from the corporate operational system. To do this, and in order to avoid interferences in the operational and functional processes of the company and to avoid congestion problems in the central system when performing operations with data, a replica of the original database is performed in our own system. To implement our data warehouse we have chosen Microsoft SQL Server. This decision was principally taken due to the availability of Microsoft Business Intelligence tools and furthermore, due to performance issues because of the high volume of data.

The corporate operational system maintains raw data from the vehicles in a non- relational (CouchDB) database. Detailed information of the CouchDB database in the central system is copied in a replica deployed in the analytic system. Later, the data from the replica is stored in our data warehouse with a specific ETL (Extract Transform and Load) module. The specific ETL module also retrieves information from summarized data which is stored in a PostgreSQL database. These summaries are enriched with details provided by transport companies, such as the information on the people that were driving the vehicles at a certain time. This information is a unique identifier assigned by the transport company to each driver with the aim of maintaining the privacy of the drivers. A detailed description of the architecture can be found in our previous work [27].

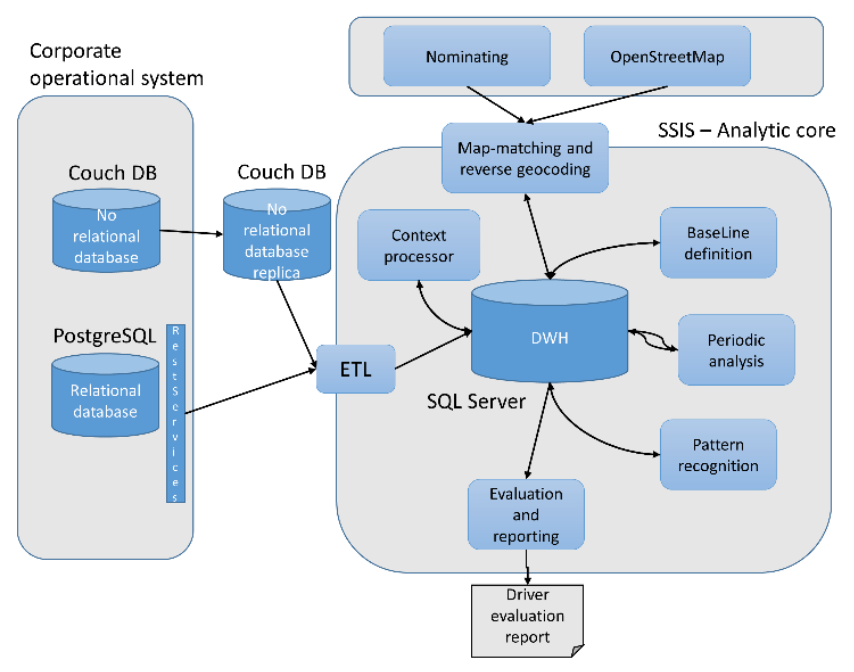

Fig. 3. Analytic system design

Once the data has been stored in our system, a set of additional modules carry out actions in order to complete the learning evaluation process. We have used the Microsoft Business Intelligent tool, SSIS (SQL Server Integration Services) to process the information and extract the driving patterns from the data gathered from the vehicles. The following paragraphs summarize the main characteristics of each module.

\section{A. Map-matching and Reverse Geocoding}

Map-matching and a reverse geocoding module performs tasks to correct latitude and longitude positions of the processed data and to translate these pair values into the nominal value of the street, respectively. Our map-matching module is implemented using an external service, constituted by a set of 
functions using the API for GPS traces in OpenStreetMap ${ }^{1}$. When the map-matching process ends, we can associate the new corrected positions with the corresponding remaining data at that location (rpms, speed, etc.). Then, once latitude and longitude pairs have been calibrated, we use Nominatim ${ }^{2}$ to perform the reverse geocoding processes. Nominatim has been installed in an Ubuntu 12.04 server and results from the conversion are loaded in the data warehouse.

\section{B. Context Processor}

The context processor module performs actions to establish the context according to the guidelines explained in Section IV.

Two processes have been designed and implemented with SSIS to characterize the context: on the one hand, a process to calculate the characteristic gearshift points for each vehicle based on the following input parameters collected from the vehicles and the on-board system: timestamp, rpm and gear.

On the other hand, a second analytical process is responsible for the evaluation of the typical idling periods for the service. Analysis of idling periods is performed by evaluating the complete fleet in order to obtain an average value of the idling pattern. Timestamp and speed are the required data to calculate the result.

Additionally, a third module, using estimations based on the available data, assesses the reliability or availability of the accessible parameters for each vehicle. Information about the available data will be used in the pattern recognition module to select the suitable configuration for processing the pattern.

Finally, results of the different context establishment modules are stored in a complementary database, along with information associated with the fleet and the vehicle.

\section{Pattern recognition, baseline definition and periodic analysis}

The pattern recognition module has been implemented using SSIS processes. This module searches among the data collected from the vehicles during a certain period, for the efficiency and inefficiency patterns described in Section IV.C. The module includes a process for each of the patterns designed, collects the necessary data from the SQL Server database and creates new tables with the detection results. From the results obtained in the detection of patterns for a certain period of time, the defined KPIs are calculated for each pattern. This period of time could belong to the baseline definition phase or to the periodic analysis phase.

To determine the presence of a certain pattern in the information collected from the vehicles, the proposed analytical system responds to a state-based model, which maintains a temporary window to determine if consecutive samples meet the conditions for a given pattern over a continuous period.

The input data needed to process the patterns vary according to the type of pattern to be analyzed and also depend on the data that may be available in the vehicle. For instance, brake switch information is not always available in certain vehicles. If this parameter is present in the input data, the quality of the results will improve, so the system will use it when it is available, although it is not indispensable to evaluate the patterns. The most relevant input data for each of the patterns are specified in Table I. In addition, the whole analysis process requires information about the timestamp and geolocation associated with the data.

\begin{tabular}{ll}
\multicolumn{1}{c}{ TABLE. I. INPUT DATA ON PATTERN RECOGNITION MODULE } \\
\hline Inertia & $\begin{array}{l}\text { Option 1) Timestamp, consumption, speed and brake (if } \\
\text { available) } \\
\text { Option 2) Timestamp, accelerator pedal position, } \\
\text { acceleration of vehicle, speed, gear and brake (if available) }\end{array}$ \\
\hline $\begin{array}{l}\text { Acceleration- } \\
\text { brake }\end{array}$ & $\begin{array}{l}\text { Timestamp, acceleration, consumption, speed and brake (if } \\
\text { available) }\end{array}$ \\
\hline $\begin{array}{l}\text { Brake- } \\
\text { acceleration }\end{array}$ & $\begin{array}{l}\text { Timestamp, acceleration, consumption, speed and brake (if } \\
\text { available) }\end{array}$ \\
\hline Idling & Timestamp, speed \\
\hline
\end{tabular}

When a pattern is detected, the system registers the event (identifying the driver and the vehicle) with the corresponding representative data and also including the KPIs for each pattern. Detailed information about the output data recorded for each pattern is shown in Table II and KPIs for each pattern are underlined. All this information is loaded in the database.

As previously mentioned, all the necessary data to extract the driving patterns are gathered from the on-board device (Section IV.A). During the baseline period, the drivers are not aware that they are being monitored. Thus, from the driving patterns of the baseline period, we obtain the initial KPI values which serve as a starting point of comparison. All drivers have their own baseline, obtained from the initial analysis. The results stored on the generated tables will be consulted in the future, to generate summary indicators that characterize this period. Subsequent analysis periods follow the same guidelines and summary indicators will be compared with the baseline indicators in order to evaluate the learning process. However, unlike during the baseline period, in subsequent periods the drivers are aware of the monitoring process.

\begin{tabular}{ll}
\multicolumn{1}{c}{ TABLE II. OUTPUT DATA ON PATTERN RECOGNITION MODULE } \\
\hline \hline Inertia & $\begin{array}{l}\text { Id driver, id vehicle, date, period of time, duration, start and } \\
\text { end geolocation points, start and end speed, duration of } \\
\text { braking (if available) }\end{array}$ \\
\hline $\begin{array}{l}\text { Acceleration- } \\
\text { brake }\end{array}$ & $\begin{array}{l}\text { Id driver, id vehicle, date, period of time, duration, start and } \\
\text { end geolocation points, start and end speed, duration of } \\
\text { braking (if available) }\end{array}$ \\
\hline $\begin{array}{l}\text { Brake- } \\
\text { acceleration }\end{array}$ & $\begin{array}{l}\text { Id driver, id vehicle, date, period of time, duration, start and } \\
\text { end geolocation points, start and end speed }\end{array}$ \\
\hline Idling & $\begin{array}{l}\text { Id driver, id vehicle, date, period of time, duration and } \\
\text { geolocation }\end{array}$ \\
\hline \hline
\end{tabular}

\section{Learning evaluation and Reporting Module}

Finally, the evaluation and reporting modules carry out the analysis of the learning process and the creation of the summary of the results respectively. The process analyses all the data mentioned previously, using for reference all the baseline data. This analysis varies in accordance with each pattern that is evaluated, depending on the individual KPIs of each pattern (duration, average speed, ...) and the dimensions in which they are included (day of the week, month of the year, hour range, street, shift ...) providing enough information to evaluate the learning progress of each driver. 
All the information extracted from the analysis is represented in two ways to obtain the desired knowledge. The first way will be recording on a map the most frequent inefficient patterns localized, providing knowledge about the main points where the driver fails. The second method is based on displaying a dashboard with all the data compared, showing the results obtained through multiple graphs. The dashboard represented will vary depending on the mode in which the analysis is executed, showing comparison between drivers, baseline against actual performance, etc.

To complete the graphical display of the results, we have developed a set of tools, using scripting language that allows us to generate XML documents from information obtained from the results of the patterns in order to analyze and visualize the detected events on maps. Moreover, to integrate all the analytic modules, we have developed a tool with a graphic user interface to perform the learning analytics process following the designed methodology.

\section{CASe Study}

The proposed analytical system has been deployed in 16 different bus companies with 880 professional drivers. However, in order to perform a detailed study for this paper, we have selected one of the fleets and three of its drivers.

The selected fleet was a Spanish urban public transport company with more than 50 lines and 22 drivers participating in the efficient driving program. We selected three drivers of the same route of this company (Driver \#1, Driver \#2 and Driver \#3) to perform a complete analysis over a period of two months. The selection of the drivers from the same route is done to enrich the analysis and the discussion of the results. As explained, the analytic system to evaluate efficient driving is based on the driver's behavior and not on other parameters influenced by the characteristics of the road or other external factors. Along the analysis period, the selected drivers covered a total distance of 2432, 2444 and $2085 \mathrm{~km}$ respectively, during 198, 182.5 and 177 driving hours respectively. Driver \#1 and Driver \#2 do the afternoon shift, while Driver \#3 does the morning shift. We apply our learning analytic process described in Section IV in order to analyze the learning progress of the selected drivers during the time period described. To analyze the evolution in the learning of efficient driving, the vehicles include the installation of the monitoring device to gather data from the ECU every 1.5 seconds. As explained in Section V, the information is stored in a data warehouse and completed with geolocation information and other context information. Then, once the information is ready after all the necessary transformations, we proceed to calculate the driving patterns. These transformations include the moving from a non-relational database to a relational database, using specific ETL modules. From the obtained results, expressed in terms of the KPIs described for each pattern, the trainer can check the learning evolution of the drivers, as well as those concepts or attitudes related to efficient driving that are not being applied correctly.

\section{A. Phase I}

Following the designed learning analytic process, we have characterized the context. All the types of buses are the same (Mercedes Citaro Euro V) and have 4 speed automatic gearboxes and run on diesel engines. To complete the characterization of the context, we have also determined gearshift points and typical idling periods. For this type of service, an urban public transport company, the average duration of idling time is 19 seconds. Regarding the availability of data, vehicles provide brake switch information.

\section{B. Phase II}

To check the evolution of the drivers during the learning process, we have established an initial period of one month for the baseline definition phase. During the baseline definition phase, the driver's activity is monitored in order to extract the final KPI results for each pattern and no feedback information is facilitated to the drivers. Results of the efficiency and inefficiency patterns (in terms of KPIs) for the baseline phase are summarized in Table III.

TABLE III. KPI RESULTS OF THE BASELINE DEFINITION PHASE

\begin{tabular}{|c|c|c|c|c|}
\cline { 2 - 5 } \multicolumn{1}{c|}{} & All drivers & Driver \#1 & Driver \#2 & Driver \#3 \\
\hline Inertia (\% time) & $20.82 \%$ & $15.73 \%$ & $21.73 \%$ & $23.05 \%$ \\
\hline AB $($ events/100Km) & $13.32 \%$ & $9.23 \%$ & $13.05 \%$ & $9.98 \%$ \\
\hline BA (events/100Km) & 327.32 & 328.32 & 372.89 & 351.2 \\
\hline
\end{tabular}

According to the results of the context analysis phase (Phase I), all idling values below 19 seconds will not be considered in the study as these idling cases are inherent to the service and traffic conditions.

Finally, after the first month of gathering data without any type of feedback to drivers, a seminar is performed by an expert in efficient driving.

\section{Phase III}

After the baseline definition phase, we have applied our analytical process to find the designed patterns for each driver among data collected from the vehicles during the following months. Table IV shows the results of the periodic analysis phase during the second month of the analysis.

TABLE IV. KPI RESULTS OF THE PERIODIC ANALYSIS PHASE FOR ONE MONTH

\begin{tabular}{|c|c|c|c|c|}
\cline { 2 - 5 } \multicolumn{1}{c|}{} & All drivers & Driver \#1 & Driver \#2 & Driver \#3 \\
\hline Idling (\% time) & $19.38 \%$ & $19.20 \%$ & $19.99 \%$ & $25.26 \%$ \\
\hline Inertia $(\%$ time $)$ & $11.59 \%$ & $9.85 \%$ & $11.47 \%$ & $10.25 \%$ \\
\hline AB $($ events/100Km) & 263.47 & 322.74 & 329.97 & 351.1 \\
\hline$B A($ events/100Km) & 68.72 & 98.94 & 75.72 & 108.11 \\
\hline
\end{tabular}

\section{Phase IV}

The analysis of the information collected during Phases II and III will provide detailed information about the learning process of each driver in order to implement the necessary actions in each case (encourage the acquisition of new efficient driving habits, reinforce practical aspects that the drivers are reluctant to apply, etc.). The following paragraphs discuss in detail the analysis of the learning process for the drivers selected for this case study.

Table $\mathrm{V}$ shows the rate of variation of each pattern, taking into account individual baseline results and monthly results. 
TABLE V. RATE OF VARIATION FOR EACH PATTERN

\begin{tabular}{|c|c|c|c|c|}
\cline { 2 - 6 } \multicolumn{1}{c|}{} & All drivers & Driver \#1 & Driver \#2 & Driver \#3 \\
\hline Idling & $-1.44 \%$ & $+3.47 \%$ & $-1.74 \%$ & $+2.21 \%$ \\
\hline Inertia & $-1.73 \%$ & $+0.62 \%$ & $-1.58 \%$ & $+0.27 \%$ \\
\hline$A B$ & $-19.51 \%$ & $-1.69 \%$ & $-11.51 \%$ & $-0.03 \%$ \\
\hline$B A$ & $-7.65 \%$ & $+25.69 \%$ & $-2.35 \%$ & $+29.86 \%$ \\
\hline
\end{tabular}

Considering the overall performance of all drivers, the general tendency is to reduce the occurrence of the patterns, thus improving efficiency. However, the appearance of inertia pattern decreases, resulting in a negative evolution of the ecodriving behavior. In addition, Driver \#1 and Driver \#3 increase the apparition of idling and BA patterns, worsening in both characteristics of efficient driving.

After a first insight into the results, an in-depth analysis is carried out with the aim of pinpointing the specific improvement points for each driver. The analysis has been divided according to the different efficiency patterns, and is detailed below.

\section{1) Idling}

Comparing the results of Tables III and IV for the idling pattern, it is noted that Driver \#1 performs much better than Driver \#2, Driver \#3 and the average in this pattern, mainly during the baseline definition phase. In the second month of the analysis, the record for Driver \#1 is much worse (increased by $3.47 \%$ ) but even in that case Driver \#1 remains below the average, while Driver \#2 improves almost $2 \%$ but is still above the mean values, and Driver \#3 obtains a negative evolution.

A detailed analysis will include the evaluation of idling periods relative to the time of the day. The selected drivers work in the afternoon shift, Driver \#1 from 14 to 22 hours and Driver \#2 from 15 to 23 hours. In addition, we have also included Driver \#3 from the morning shift. Fig. 4 shows the idling periods distributed according to the time of the day.

An interesting result is that the tendency is the same for all drivers depending on the time. Thereby, Fig. 4 shows how the hourly fluctuation has the same sign (with the exception of Driver \#2 at 16 hours) in the cases shown (Drivers \#1, \#2 and \#3, and the average).

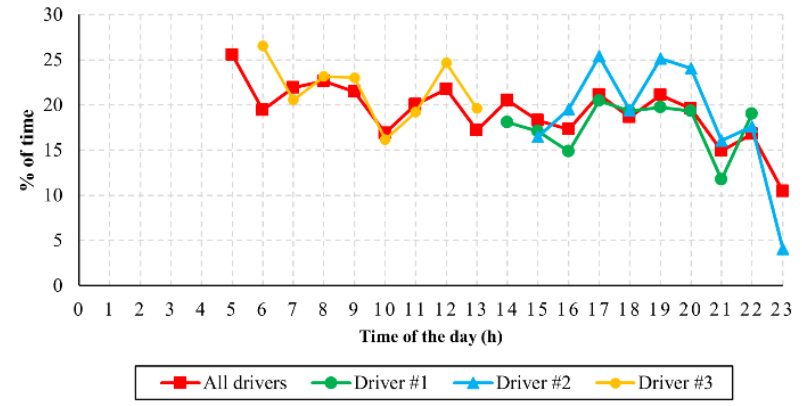

Fig. 4. Idling pattern: KPI (\% of time) results by hours

This study illustrates how a particular driver performs better or worse in relation to the traffic or the number of passengers. For instance, we can see how Driver \#2 increases his idling time much more than his colleagues during rush hours, yet he is near to the average when everything is less busy.

In conclusion, Driver \#2 shows a better evolution, meaning that the efficient driving learning approach has influenced his behavior. However, the percentage of time of the idling pattern based on the time of the day shows higher values for Driver \#2 because, in general, he has longer idling periods. According to the learning evaluation process, we are able to perform a detailed analysis of the idle pattern for Driver \#2 in order to identify the cause of his performance. A detailed analysis of the geographic location for idle values beyond 2 minutes reveals that these points are concentrated in the end of the route. Therefore, Driver \#2 does not turn off the engine at the end of the route, so he registers longer periods of idling than the rest of the drivers, despite being the same route. In conclusion, successive reinforcement reports for Driver \#2 should emphasize the recommendation of turning off the engine during long periods of idling, but highlighting that positive results are being reached.

\section{2) Inertia}

Driver \#1 and \#3 have a slight improvement in the inertia pattern. On the contrary, Driver \#2 evolves in a similar way to the whole group, as he decreases inertia time and maintains the distance below the mean values.

At first glance these data could seem to suggest a better performance by Driver \#2, as he generates more inertia time. However, Driver \#1 and \#3 perform better, as they managed to improve $0.62 \%$ and $0.27 \%$ while the fleet tendency during the analyzed period was to worsen by $1.73 \%$. Therefore, as explained previously, the evaluation process is based on individual assessment and thus, Driver \#2 shows a negative evolution in the inertia pattern: he decreases the inertia time from one month to the next.

The hourly evolution analysis shows interesting data. Fig. 5 shows how the records in the first half of the day are much better than those in the second half. Therefore, both drivers have more difficulty in developing inertia than those working during the morning. We can see how performances before 3 p.m. manage to obtain much better inertia records than in the afternoon/evening. The explanation is that, with a higher density of traffic in the morning, the driver will be braking longer than in the afternoon, so the time with no fuel consumption increases, causing more periods of inertia. Note that the inertia periods that include the use of the brakes are related with the movement of the vehicle with null consumption and not with harsh braking events. However, the selected driver of the morning shift (Driver \#3) performs worse in the inertia pattern than the rest of the fleet. Nevertheless, Driver \#3 obtains a positive individual evolution in the inertia pattern (Table V).

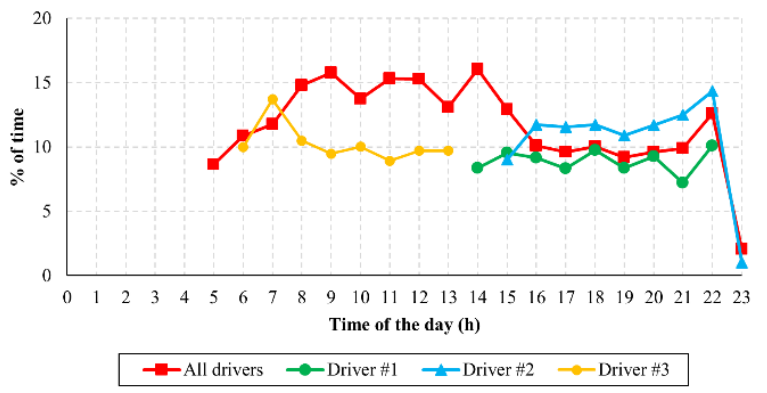

Fig. 5. Inertia pattern: KPI (\% of time) results by hours 


\section{3) Acceleration - Brake (AB)}

In this case, drivers improve their $\mathrm{AB}$ performance (Table $\mathrm{V})$, as is the tendency of the whole group of drivers. This reduction in the detection of the $\mathrm{AB}$ pattern, from the baseline period to the next month of analyzed data, can be graphically observed in Fig. 6. Fig. 6 shows concentration points for the AB pattern in a specific area of the city of the case study. As previously defined, the $A B$ pattern includes events in which the duration is less than 2 seconds, that means the time between the driver stopping accelerating until there is a sudden harsh braking. The $A B$ events registered during the baseline period are represented by black points and the $\mathrm{AB}$ events during the following month of the analysis are summarized using white points. We can see how these points are gathered mainly around bus stops (marked with a black circle) and how the incidence of this pattern diminishes in the second month of the analysis (white points) in relation to the baseline definition phase (black points).

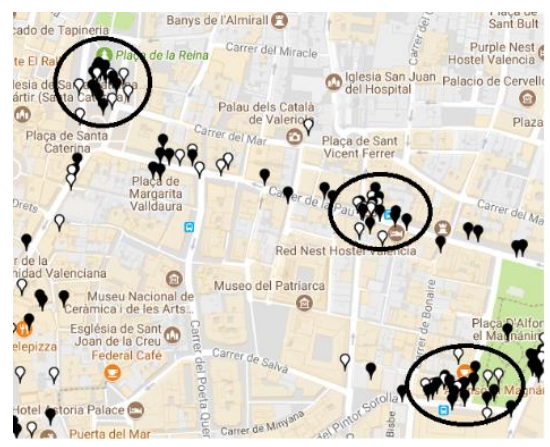

Fig. 6. AB pattern concentration points

Driver \#2 improves in a similar way to the average of all drivers (42.92 events/100km against 63.85) while Drivers \#1 and \#3 only show a slight improvement. To analyze this behavior in detail, we have carried out an evaluation of both drivers of the afternoon shift (Driver \#1 and \#2), comparing results of the number of $\mathrm{AB}$ events per time of the day for each month.

Fig. 7 shows the results for Driver \#1 and Driver \#2. According to the analyzed data, Driver \#1 would have shown worse records in the second month without the big improvement between 21 and 22 hours, as the rest of the periods were quite balanced, with a couple of clear increases at 15 and 18 hours (the one at 13 hours is not relevant, as it is 1 event in $0.83 \mathrm{~km}$, making 120 events $/ 100 \mathrm{~km}$ ). On the contrary, Driver \#2 has a global improvement with an exception at 16 hours, indicating a more solid performance and a much better result in this pattern.

4) Brake - Acceleration (BA)

This pattern, due to its nature, tends to produce worse results when the traffic is dense, as vehicles run closer together and distances are reduced between them. Thus, we have identified more harsh-braking events followed by the acceleration of the vehicle as the main indicative of the driving behavior which does not maintain the safety distance during rush hours. For this reason, an increase of BA events $/ 100 \mathrm{Km}$ can be observed in Fig. 8 during rush hours in the morning (8-9 a.m and 11-12 a.m). In the afternoon, there is a slight increase at $17 \mathrm{~h}$. However, both of the studied drivers obtain records over the average. While Driver \#2 has acceptable values even when over the average (Tables III and IV), Driver \#1 experiences a relevant increase of BA events in the second month (Table V), leading us to think that the driver has fallen into some negligence or relaxed attitude that has to be corrected.
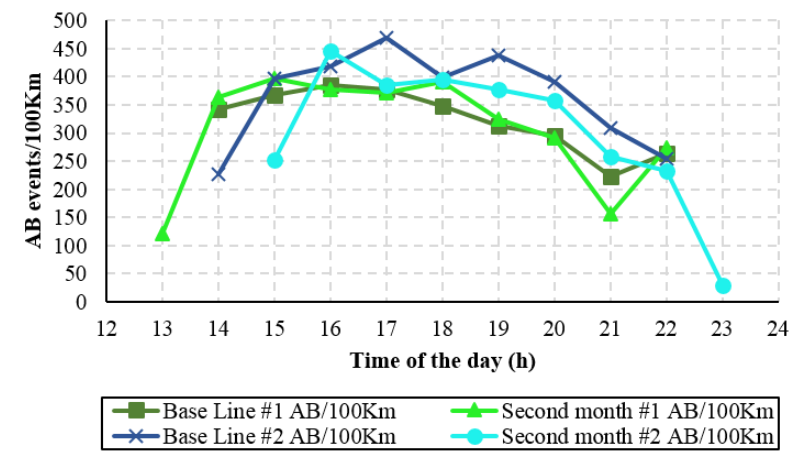

Fig. 7. AB pattern: KPI (events/100Km) results for Driver \#1 and Driver \#2

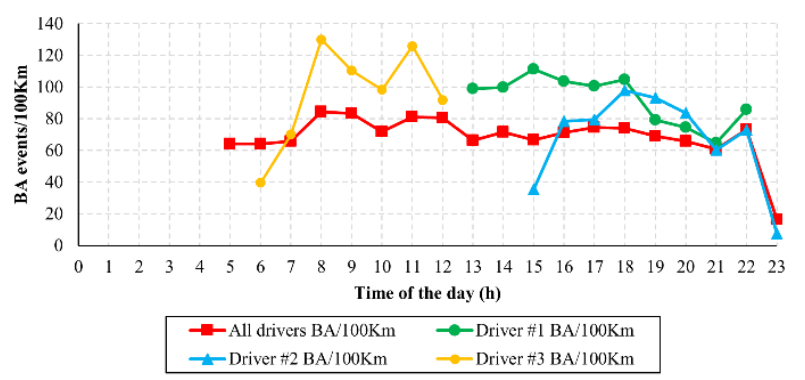

Fig. 8. BA pattern: KPI (events/100Km) results by hours

According to the hourly distribution graphic (Fig. 8), Drivers $\# 1$, \#2 and \#3 perform worse than the average of the complete fleet. If we analyze Fig. 8, Driver \#3, from the morning shift, performs worse during rush hours in the morning (8 a.m and 11 a.m) and Driver \#1, from the afternoon shift, performs much better after 19 hours. This situation could indicate that high traffic density affects the driving behavior of Driver \#1 and \#3. The opposite effect is found in the behavior of Driver \#2. Moreover, Driver \#1 and \#2 increase BA events at 22 hours, where vehicles are returned to the garage without passengers and drivers do not maintain the same good driving practices as when passengers are on board.

Table V summarizes the evolution of the drivers regarding all of the four patterns. Results of the evaluation show that Driver \#1 and \#3 have a negative evolution in the idling and BA patterns. However, a slight improvement in inertia and $A B$ patterns is detected, probably due to the extra use of brakes in the second month, as stated in pattern BA. Regarding Driver \#2, results only worsen in inertia, improving in the other areas of efficient and safe driving. As a result, individual and personalized formative actions would be applied in order to achieve a more efficient activity and a safer work environment, taking into account not only inefficient actions but also conflictive areas of the city or time of the day. Therefore, 
drivers receive individualized recommendations and their motivation in the learning process in efficient driving increases, with the consequent positive evolution in the long-term.

Finally, as stated in Section "I. Introduction", the information obtained from the analysis of the driver's evolution can be used in a reward program. In this sense, the fleet manager should detail the basis of such a system, and the duration of the period to obtain the classification. In this paper, we focus on the analytic system to evaluate driving efficiency and the case study reviews the driver's evolution over a period of two months. Table $\mathrm{V}$ shows that Driver \#2 improves his performance in idling, AB and BA patterns, while Driver \#1 and Driver \#3 only show a positive evolution in the inertia and $\mathrm{AB}$ patterns. Therefore, Driver \#2 evolves in more aspects than the rest of the drivers. This information is the basis of the reward system but other information is used to enrich the distribution of the drivers through the ranking, such as the efficiency level based on time of the day, day of the week or vehicle.

In order to verify the evolution in driving efficiency, we analyze the drivers' behavior after 6 months. The monthly feedback process is aimed at gradually improving the efficient driving behavior, from the basic driving patterns to the more complex patterns. With this idea, the initial drivers' feedback reports will focus on improving inertia and idling, since the $\mathrm{AB}$ and $\mathrm{BA}$ patterns are technically more complex.

Table VI summarizes the inertia KPI values after 6,7 and 8 months. Driver \#3 continues improving in the inertia pattern over the months. Driver \#1 shows a great increase in the inertia performance except for a slight deterioration in the last month. Finally, Driver \#2 (who shows the best performance during the baseline period) improves the inertia pattern from the second month, except during the last analyzed month which shows a minor decline.

TABLE VI. INERTIA KPI RESUlts (\% TIME) OF THE PERIODIC ANALYSIS PHASE

\begin{tabular}{|c|c|c|c|}
\cline { 2 - 4 } \multicolumn{1}{c|}{} & Driver \#1 & Driver \#2 & Driver \#3 \\
\hline Baseline & $9.23 \%$ & $13.05 \%$ & $9.98 \%$ \\
\hline Second month & $9.85 \%$ & $11.47 \%$ & $10.25 \%$ \\
\hline Sixth month & $13.24 \%$ & $12.57 \%$ & $10.96 \%$ \\
\hline Seventh month & $13.88 \%$ & $12.82 \%$ & $11.01 \%$ \\
\hline Eighth month & $13.42 \%$ & $12.73 \%$ & $11.44 \%$ \\
\hline
\end{tabular}

Since Driver \#2 begins with a good inertia performance in the baseline period, we check his evolution in the idle pattern. Table VII shows the results. Apparently, the feedback report has not had the desired effect to improve this behavior as Driver \#2 increases the KPI values (and should decrease for the idle pattern).

TABLE VII. IDLE KPI RESULTS (\% TIME) OF THE PERIODIC ANALYSIS PHASE

\begin{tabular}{|c|c|}
\cline { 2 - 2 } \multicolumn{1}{c|}{} & Driver \#2 \\
\hline Baseline & $21,73 \%$ \\
\hline Second month & $19,99 \%$ \\
\hline Sixth month & $22.08 \%$ \\
\hline Seventh month & $23.76 \%$ \\
\hline Eighth month & $23.23 \%$ \\
\hline
\end{tabular}

However, the analysis of idle periods of more than 2 minutes (the most inefficient) show that the worse value is reached during the baseline period, in which no feedback is provided. During the second month, this value is reduced to $0.26 \%$. In the months 6,7 and 8 , the values fluctuate, indicating that, for this driver, the recommendation to turn off the engine in stops of more than two minutes must always be present in the feedback reports as a reminder since, otherwise, the performance deteriorates.

TABLE VIII. IDLE KPI RESULTS (\% TIME) FOR IDLING TIME > 120 SECONDS

\begin{tabular}{|c|c|}
\cline { 2 - 2 } \multicolumn{1}{c|}{} & Driver \#2 \\
\hline Baseline & $0.97 \%$ \\
\hline Second month & $0.26 \%$ \\
\hline Sixth month & $0.77 \%$ \\
\hline Seventh month & $0.57 \%$ \\
\hline Eighth month & $0.78 \%$ \\
\hline
\end{tabular}

The reward system has been implemented in two of the professional bus companies from our analytic system. In one of the fleets, the prize consists of a week's cruise. In the other fleet, the prize includes two days of expert driving courses on a professional circuit. However, the evaluation in the long-term of the reward system should be addressed in our future work.

\section{CONCLUSIONS AND FUTURE WORK}

In this paper, we propose a learning analytic system, which improves a previous efficient driving learning methodology already in use in 16 different companies with 880 professional drivers. We have demonstrated that the proposed novel method to evaluate individual driver's performance, based on a set of designed patterns related to efficiency, security and anticipation behaviors, can accurately describe the learning process in efficient driving. In addition, inefficient actions can be located depending on the zone, street or even the time of day, thus facilitating the detailed description of the feedback needed to correct these behaviors.

Results obtained from a real learning analysis show that the system is able to identify efficient and inefficient actions, which are the basis for evaluating the improvement of the driver's behavior over time. We have also verified that our system provides vital information to schedule future formative and corrective actions and measure their effectiveness. This is a key issue for a lifelong learning process based on continuous evaluation in which it is necessary to know the situations that have to be corrected. Moreover, the accurate indicators obtained during the evaluation phase are decisive in the rewarding program due to the fact that drivers are more involved during the efficient driving learning process, as the system can identify when, where and how they can improve their behavior. Results show that, in general terms, drivers evolve positively after few months of being part of the efficient driving learning program.

The paper shows the need for such systems to control the learning processes while the drivers are actually working. In these scenarios, an evaluation based on specific tests does not guarantee that those skills are actually used in the work activity. Our analytic system to evaluate efficient driving and its complexity is transparent for the drivers and does not interfere in their normal work activity. This characteristic means that our system has been widely adopted in professional fleets.

Future work will focus on the evaluation of the reward program in the long-term and the inclusion of the analytical 
system into an adaptive learning process. In this new approach, personalized recommendations and customized training plans will be designed for each driver, using the information produced in the learning analytic system. Moreover, an automatic recommendation system will be introduced in the learning analytic system to produce a revision of the drivers' performance with minimum participation of the trainers. In addition, we will continue to design new patterns to detect further efficient and inefficient behaviors, with the aim of covering all situations while driving. Finally, our future work will include a socio-demographic analysis of the influence of elements such us age or driving experience on the adoption of efficient driving behavior and the inclusion of more data sources, such as traffic and road conditions to improve the detailed results on efficient driving.

\section{REFERENCES}

[1] International Transport Forum, Ed., 'Drop in global travel volume leads to cut in transport emissions'. May-2010.

[2] European Commision, 'White Paper: Roadmap to a Single European Transport Area - Towards a competitive and resource efficient transport system'. 2011.

[3] T. Onoda, 'IEA policies - G8 recommendations and an afterwards', Energy Policy, vol. 37, no. 10, pp. 3823-3831, Oct. 2009.

[4] A. E. af Wåhlberg, 'Long-term effects of training in economical driving: Fuel consumption, accidents, driver acceleration behavior and technical feedback', International Journal of Industrial Ergonomics, vol. 37, no. 4, pp. 333-343, Apr. 2007.

[5] A. Rionda, X. G. Pañeda, R. García, G. Díaz, D. Martínez, M. Mitre, D. Arbesú, and I. Marín, 'Blended learning system for efficient professional driving', Computers \& Education, vol. 78, pp. 124-139, Sep. 2014.

[6] J. N. Barkenbus, 'Eco-driving: An overlooked climate change initiative', Energy Policy, vol. 38, no. 2, pp. 762-769, Feb. 2010

[7] C. Andrieu and G. S. Pierre, 'Comparing Effects of Eco-driving Training and Simple Advices on Driving Behavior', Procedia - Social and Behavioral Sciences, vol. 54, pp. 211-220, Oct. 2012.

[8] M. J. M. Sullman, L. Dorn, and P. Niemi, 'Eco-driving training of professional bus drivers - Does it work?', Transportation Research Part C: Emerging Technologies, vol. 58, Part D, pp. 749-759, 2015.

[9] M. Zarkadoula, G. Zoidis, and E. Tritopoulou, 'Training urban bus drivers to promote smart driving: A note on a Greek eco-driving pilot program', Transportation Research Part D: Transport and Environment, vol. 12, no. 6, pp. 449-451, Aug. 2007.

[10] H. K. Strömberg and I. C. M. Karlsson, 'Comparative effects of ecodriving initiatives aimed at urban bus drivers - Results from a field trial', Transportation Research Part D: Transport and Environment, vol. 22, pp. 28-33, Jul. 2013.

[11] M. Rutty, L. Matthews, J. Andrey, and T. D. Matto, 'Eco-driver training within the City of Calgary's municipal fleet: Monitoring the impact', Transportation Research Part D: Transport and Environment, vol. 24, pp. 44-51, Oct. 2013.

[12] C. Vagg, C. J. Brace, D. Hari, S. Akehurst, J. Poxon, and L. Ash, 'Development and Field Trial of a Driver Assistance System to Encourage Eco-Driving in Light Commercial Vehicle Fleets', IEEE Transactions on Intelligent Transportation Systems, vol. 14, no. 2, pp. 796-805, Jun. 2013.

[13] B. Beusen, S. Broekx, T. Denys, C. Beckx, B. Degraeuwe, M. Gijsbers, K. Scheepers, L. Govaerts, R. Torfs, and L. I. Panis, 'Using on-board logging devices to study the longer-term impact of an ecodriving course', Transportation Research Part D: Transport and Environment, vol. 14, no. 7, pp. 514-520, Oct. 2009.

[14] H. Liimatainen, 'Utilization of Fuel Consumption Data in an Ecodriving Incentive System for Heavy-Duty Vehicle Drivers', IEEE Transactions on Intelligent Transportation Systems, vol. 12, no. 4, pp. 1087-1095, Dec. 2011.

[15] J. Harvey, N. Thorpe, and R. Fairchild, 'Attitudes towards and perceptions of eco-driving and the role of feedback systems', Ergonomics, vol. 56, no. 3, pp. 507-521, 2013.
[16] B. Caulfield, W. Brazil, K. Ni Fitzgerald, and C. Morton, 'Measuring the success of reducing emissions using an on-board eco-driving feedback tool', Transportation Research Part D: Transport and Environment, vol. 32, pp. 253-262, Oct. 2014.

[17] M. Sivak and B. Schoettle, 'Eco-driving: Strategic, tactical, and operational decisions of the driver that influence vehicle fuel economy', Transport Policy, vol. 22, pp. 96-99, Jul. 2012.

[18] K. Boriboonsomsin, A. Vu, and M. Barth, 'Eco-Driving: Pilot Evaluation of Driving Behavior Changes among U.S. Drivers', UCTC Research Paper, Aug. 2010.

[19] C. Rolim, P. Baptista, G. Duarte, T. Farias, and Y. Shiftan, 'Quantification of the Impacts of Eco-driving Training and Real-time Feedback on Urban Buses Driver's Behaviour', Transportation Research Procedia, vol. 3, pp. 70-79, 2014.

[20] M. Staubach, N. Schebitz, F. Köster, and D. Kuck, 'Evaluation of an eco-driving support system', Transportation Research Part F: Traffic Psychology and Behaviour, vol. 27, Part A, pp. 11-21, Nov. 2014

[21] V. Corcoba Magaña and M. Muñoz-Organero, 'Discovering Regions Where Users Drive Inefficiently on Regular Journeys', IEEE Transactions on Intelligent Transportation Systems, vol. 16, no. 1, pp. 221-234, Feb. 2015.

[22] J. C. Ferreira, J. de Almeida, and A. R. da Silva, 'The Impact of Driving Styles on Fuel Consumption: A Data-Warehouse-and-DataMining-Based Discovery Process', IEEE Transactions on Intelligent Transportation Systems, vol. 16, no. 5, pp. 2653-2662, Oct. 2015.

[23] L. Pozueco, A. Rionda, A.G. Pañeda, J.A. Sánchez, X.G. Pañeda, R. García, D. Melendi, A.G. Tuero et al., 'Impact of on-board tutoring systems to improve driving efficiency of non-professional drivers', IET Intelligent Transport Systems, Jan. 2017.

[24] T. Wanner and E. Palmer, 'Personalising learning: Exploring student and teacher perceptions about flexible learning and assessment in a flipped university course', Computers \& Education, vol. 88, pp. 354-369, Oct. 2015.

[25] L. Pozueco, A. G. Tuero, X. G. Pañeda, D. Melendi, R. Garcia, A. G. Pañeda, A. Rionda, G. Diaz, and M. Mitre, 'Adaptive learning for efficient driving in urban public transport', in 2015 International Conference on Computer, Information and Telecommunication Systems (CITS), 2015, pp. 1-5.

[26] D. Parmenter, 'Key performance indicators (KPI): developing, implementing, and using winning KPIs'. John Wiley \& Sons, 2010.

[27] A. G. Pañeda et al., "An Architecture for a Learning Analytics System Applied to Efficient Driving," IEEE Revista Iberoamericana de Tecnologias del Aprendizaje, vol. 11, no. 3, pp. 137-145, Aug. 2016.

Laura Pozueco is a Telecommunications Engineer from the University of Oviedo with a PhD from the Spanish University for Distance Education (UNED). Her current research interests are in the area of efficient driving.

Alejandro G. Tuero (BS in Mechanics) is a researcher specialized in machines and mechanisms and with wide knowledge in the automotive industry. He has been part of this research group since January 2015.

Alejandro G. Pañeda is a Computer Science Engineer from the University of Oviedo, with an interest in the area of datamining and big data systems.

Xabiel G. Pañeda is a Computer Science Engineer with a $\mathrm{PhD}$ from the University of Oviedo, with an interest in mobile systems, content distribution networks and efficient driving. He is an Associate Professor at the University of Oviedo.

David Melendi is a Computer Science Engineer with a $\mathrm{PhD}$ from the University of Oviedo, with an interest in multimedia systems, databases and embedded systems. He is an Associate Professor at the University of Oviedo.

Roberto García is a Telecommunications Engineer from The Technical University of Madrid with a PhD from the University of Oviedo, with an interest in telecommunication networks. He is an Associate Professor at the University of Oviedo.

Gabriel Díaz ( $\mathrm{PhD}$ in Physics) is an associate professor in the Telematics Area of Spanish University for Distance Education (UNED). He is an active researcher in e-learning systems and techniques.

Abel Rionda has an MSc in Computer Engineering from the University of Oviedo and a PhD from UNED. He is CEO of ADN Mobile Solutions and his research interests are efficient driving and embedded systems. 